\title{
Research Article \\ Optimal Control of Polymer Flooding Based on Maximum Principle
}

\author{
Yang Lei, ${ }^{1}$ Shurong Li, ${ }^{1}$ Xiaodong Zhang, ${ }^{1}$ \\ Qiang Zhang, ${ }^{1}$ and Lanlei Guo ${ }^{2}$ \\ ${ }^{1}$ College of Information and Control Engineering, China University of Petroleum, \\ Qingdao, 266580, China \\ 2 Research Institute of Geological Science, Sinopec Shengli Oilfield Company, \\ Dongying 257000, China \\ Correspondence should be addressed to Yang Lei, yutian_hdpu2003@163.com
}

Received 8 March 2012; Accepted 29 May 2012

Academic Editor: Xianxia Zhang

Copyright ( $) 2012$ Yang Lei et al. This is an open access article distributed under the Creative Commons Attribution License, which permits unrestricted use, distribution, and reproduction in any medium, provided the original work is properly cited.

\begin{abstract}
Polymer flooding is one of the most important technologies for enhanced oil recovery (EOR). In this paper, an optimal control model of distributed parameter systems (DPSs) for polymer injection strategies is established, which involves the performance index as maximum of the profit, the governing equations as the fluid flow equations of polymer flooding, and the inequality constraint as the polymer concentration limitation. To cope with the optimal control problem (OCP) of this DPS, the necessary conditions for optimality are obtained through application of the calculus of variations and Pontryagin's weak maximum principle. A gradient method is proposed for the computation of optimal injection strategies. The numerical results of an example illustrate the effectiveness of the proposed method.
\end{abstract}

\section{Introduction}

It is of increasing necessity to produce oil fields more efficiently and economically because of the ever-increasing demand for petroleum worldwide. Since most of the significant oil fields are mature fields and the number of new discoveries per year is decreasing, the use of EOR processes is becoming more and more imperative. At present, polymer flooding technology is the best method for chemically EOR [1]. It could reduce the water-oil mobility ratio and improve sweep efficiency [2-5].

Because of the high cost of chemicals, it is essential to optimize polymer injection strategies to provide the greatest oil recovery at the lowest cost. The optimization procedure involves maximizing the objective function (cumulative oil production or profit) from a polymer flooding reservoir by adjusting the injection concentration. One way of solving this problem is direct optimization with the reservoir simulator. Numerical models are 
used to evaluate the complex interactions of variables affecting development decisions, such as reservoir and fluid properties and economic factors. Even with these models, the current practice is still the conventional trial and error approach. In each trial, the polymer concentration of an injection well is selected based on the intuition of the reservoir engineer. This one-well-at-a-time approach may lead to suboptimal decisions because engineering and geologic variables affecting reservoir performance are often nonlinearly correlated. And the problem definitely compounds when multiple producers and injectors are involved in a field development case. The use of the optimal control method offers a way out.

The optimal control method has been researched in EOR techniques in recent years. Ramirez et al. [6] firstly applied the theory of optimal control to determine the best possible injection strategies for EOR processes. Their study was motivated by the high operation costs associated with EOR projects. The objective of their study was to develop an optimization method to minimize injection costs while maximizing the amount of oil recovered. The performance of their algorithm was subsequently examined for surfactant injection as an EOR process in a one-dimensional core flooding problem [7]. The control for the process was the surfactant concentration of the injected fluid. They observed a significant improvement in the ratio of the value of the oil recovered to the cost of the surfactant injected from 1.5 to about 3.4. Optimal control was also applied to steam flooding by Liu et al. [8]. They developed an approach using optimal control theory to determine operating strategies to maximize the economic attractiveness of steam flooding process. Their objective was to maximize a performance index which is defined as the difference between oil revenue and the cost of injected steam. Their optimization method also obtained significant improvement under optimal operation. Ye et al. [9] were involved in the study of optimal control of gas-cycling in condensate reservoirs. It was shown that both the oil recovery and the total profit of a condensate reservoir can be enhanced obviously through optimization of gas production rate, gas injection rate, and the mole fractions of each component in injection gas. Daripa et al. [10-15] researched the basic physical mechanisms that contribute to poor oil recovery by EOR technologies and how to individually control each of these physical mechanisms. Brouwer and Jansen [16] and Sarma et al. [17] used the optimal control theory as an optimization algorithm for adjusting the valve setting in smart wells of water flooding. The water flooding scheme that maximized the profit was numerically obtained by combining reservoir simulation with control theory practices of implicit differentiation. They were able to achieve improved sweep efficiency and delayed water breakthrough by dynamic control of the valve setting.

For the previous work on optimal control of polymer flooding, Guo et al. [18] applied the iterative dynamic programming algorithm to solve the OCP of a one-dimensional core polymer flooding. However, the optimal control model used in their study is so simple that it is not adapted for practical oilfield development. As a result of the complicated nature of reservoir models with nonlinear constraints, it is very tedious and troublesome to cope with a large number of grid points for the state variables and control variables. To avoid these difficulties, Li et al. [19] and Lei et al. [20] used the genetic algorithms to determine the optimal injection strategies of polymer flooding and the reservoir model equations were treated as a "black box." The genetic algorithms are capable of finding the global optimum on theoretical sense, but, as Sarma et al. [17] point out, they require tens or hundreds of thousand reservoir simulation runs of very large model and are not able to guarantee monotonic maximization of the objective function.

In this paper, an optimal control model of DPS for polymer flooding is established which maximizes the profit by adjusting the injection concentration. Then the determination 
of polymer injection strategies turns to solve this OCP of DPS. Necessary conditions for optimality are obtained by Pontryagin's weak maximum principle. A gradient numerical method is presented for solving the OCP. Finally, an example of polymer flooding project involving a heterogeneous reservoir case is investigated and the results show the efficiency of the proposed method.

\section{Mathematical Formulation of Optimal Control}

\subsection{Performance Index}

Let $\Omega \in R^{2}$ denote the domain of reservoir with boundary $\partial \Omega, n$ be the unit outward normal on $\partial \Omega$, and $(x, y) \in \Omega$ be the coordinate of a point in the reservoir. Given a fixed final time $t_{f}$, we set $\Psi=\Omega \times\left[0, t_{f}\right], \Gamma=\partial \Omega \times\left[0, t_{f}\right]$, and suppose that there exist $N_{w}$ injection wells and $N_{o}$ production wells in the oilfield. The injection and production wells are located at $L_{w}=\left\{\left(x_{w i}, y_{w i}\right) \mid i=1,2, \ldots, N_{w}\right\}$ and $L_{o}=\left\{\left(x_{o j}, y_{o j}\right) \mid j=1,2, \ldots, N_{o}\right\}$, respectively. This descriptive statement of the cost functional must be translated into a mathematical form to use quantitative optimization techniques. The oil value can be formulated as

$$
\int_{0}^{t_{f}} \iint_{\Omega} \xi_{o}\left(1-f_{w}\right) q_{\text {out }} d \sigma d t
$$

where $\xi_{o}$ is the cost of oil per unit mass $\left(10^{4} \$ / \mathrm{m}^{3}\right), f_{w}(x, y, t)$ is the fractional flow of water, and $q_{\text {out }}(x, y, t)$ is the flow velocity of production fluid (m/day). We define $q_{\text {out }}(x, y, t) \geq 0$ at $(x, y) \in L_{o}$ and $q_{\text {out }}(x, y, t) \equiv 0$ at $(x, y) \notin L_{o}$.

The polymer cost is expressed mathematically as

$$
\int_{0}^{t_{f}} \iint_{\Omega} \xi_{p} q_{\text {in }} c_{\text {pin }} d \sigma d t
$$

where $\xi_{p}$ is the cost of oil per unit volume $\left(10^{4} \$ / \mathrm{m}^{3}\right), c_{\mathrm{pin}}(x, y, t)$ is the polymer concentration of the injection fluid $(\mathrm{g} / \mathrm{L})$, and $q_{\text {in }}(x, y, t)$ is the flow velocity of injection fluid (m/day). We define $q_{\text {in }}(x, y, t) \geq 0$ at $(x, y) \in L_{w}$ and $q_{\text {in }}(x, y, t) \equiv 0$ at $(x, y) \notin L_{w}$.

The objective functional is, therefore,

$$
\max J=\int_{0}^{t_{f}} \iint_{\Omega}\left[\xi_{o}\left(1-f_{w}\right) q_{\mathrm{out}}-\xi_{p} q_{\mathrm{in}} c_{\mathrm{pin}}\right] d \sigma d t
$$

\subsection{Governing Equations}

The maximization of the cost functional $J$ given by (2.3) is not totally free but is constrained by the system process dynamics. The governing equations of the polymer flooding process must therefore be developed to describe the flow of both the aqueous and oil phases through the porous media of a reservoir formation. The equations used in this paper allow for the adsorption of polymer onto the solid matrix in addition to the convective and dispersive mechanisms of mass transfer. Let $p(x, y, t), S_{w}(x, y, t)$ and $c_{p}(x, y, t)$ denote the pressure, water saturation, and polymer concentration of the oil reservoir, respectively, at a point 
$(x, y) \in \Omega$ and a time $t \in\left[0, t_{f}\right]$, then $p(x, y, t), S_{w}(x, y, t)$, and $c_{p}(x, y, t)$ satisfy the following partial differential equations (PDEs).

(i) The flow equation for oil phase

$$
\frac{\partial}{\partial x}\left(k_{p} r_{o} \frac{\partial p}{\partial x}\right)+\frac{\partial}{\partial y}\left(k_{p} r_{o} \frac{\partial p}{\partial y}\right)-\left(1-f_{w}\right) q_{\mathrm{out}}=h \frac{\partial a_{o}}{\partial t}, \quad(x, y, t) \in \Psi
$$

(ii) The flow equation for water phase

$$
\frac{\partial}{\partial x}\left(k_{p} r_{w} \frac{\partial p}{\partial x}\right)+\frac{\partial}{\partial y}\left(k_{p} r_{w} \frac{\partial p}{\partial y}\right)+q_{\text {in }}-f_{w} q_{\text {out }}=h \frac{\partial a_{w}}{\partial t}, \quad(x, y, t) \in \Psi
$$

(iii) The flow equation for polymer component

$$
\begin{aligned}
& \frac{\partial}{\partial x}\left(k_{d} r_{d} \frac{\partial c_{p}}{\partial x}\right)+\frac{\partial}{\partial x}\left(k_{p} r_{c} \frac{\partial p}{\partial x}\right)+\frac{\partial}{\partial y}\left(k_{d} r_{d} \frac{\partial c_{p}}{\partial y}\right)+\frac{\partial}{\partial y}\left(k_{p} r_{c} \frac{\partial p}{\partial y}\right)+q_{\text {in }} c_{\text {pin }}-f_{w} q_{\text {out }} c_{p} \\
& =h \frac{\partial a_{c}}{\partial t}, \quad(x, y, t) \in \Psi .
\end{aligned}
$$

(iv) The boundary conditions and initial conditions

$$
\begin{gathered}
\left.\frac{\partial p}{\partial n}\right|_{\partial \Omega}=0,\left.\quad \frac{\partial S_{w}}{\partial n}\right|_{\partial \Omega}=0,\left.\quad \frac{\partial c_{p}}{\partial n}\right|_{\partial \Omega}=0, \quad(x, y, t) \in \Gamma, \\
p(x, y, 0)=p^{0}(x, y), \quad S_{w}(x, y, 0)=S_{w}^{0}(x, y), \\
c_{p}(x, y, 0)=c_{p}^{0}(x, y), \quad(x, y) \in \Omega,
\end{gathered}
$$

where the corresponding parameters are defined as

$$
\begin{gathered}
k_{p}=K h, \quad k_{d}=D h, \\
r_{o}=\frac{k_{r o}}{B_{o} \mu_{o}}, \quad r_{w}=\frac{k_{r w}}{B_{w} R_{k} \mu_{w}}, \quad r_{c}=\frac{k_{r w} c_{p}}{B_{w} R_{k} \mu_{p}}, \quad r_{d}=\frac{\phi_{p} S_{w}}{B_{w}} \\
a_{o}=\frac{\phi\left(1-S_{w}\right)}{B_{o}}, \quad a_{w}=\frac{\phi S_{w}}{B_{w}}, \quad a_{c}=\frac{\phi_{p} S_{w} c_{p}}{B_{w}}+\rho_{r}(1-\phi) C_{r p} .
\end{gathered}
$$

The constant coefficient $K(x, y)$ is the absolute permeability $\left(\mu \mathrm{m}^{2}\right), h$ is the thickness of the reservoir bed $(\mathrm{m}), D$ is the diffusion coefficient of polymer $\left(\mathrm{m}^{2} / \mathrm{s}\right), \rho_{r}\left(\mathrm{~kg} / \mathrm{m}^{3}\right)$ is the rock density, and $\mu_{o}$ (mPa.s) is the oil viscosity. 
The oil volume factor $B_{o}$, the water volume factor $B_{w}$, the rock porosity $\phi$, and the effective porosity to polymer $\phi_{p}$ are expressed as functions of the reservoir pressure $p$ :

$$
\begin{gathered}
B_{o}=\frac{B_{o r}}{\left[1+C_{o}\left(p-p_{r}\right)\right]}, \\
B_{w}=\frac{B_{w r}}{\left[1+C_{w}\left(p-p_{r}\right)\right]}, \\
\phi=\phi_{r}\left[1+C_{R}\left(p-p_{r}\right)\right], \\
\phi_{p}=f_{a} \phi,
\end{gathered}
$$

where $p_{r}$ is the reference pressure (MPa), $\phi_{r}, B_{o r}$, and $B_{w r}$ denote the porosity, the oil, and water volume factor under the condition of the reference pressure, respectively, $f_{a}$ is the effective pore volume coefficient, $C_{o}, C_{w}$, and $C_{R}$ denote the compressibility factors of oil, water, and rock, respectively.

Functions relating values of the oil and water relative permeabilities $k_{r o}$ and $k_{r w}$ to the water saturation $S_{w}$ are

$$
\begin{aligned}
& k_{r w}=k_{r w r o}\left(\frac{S_{w}-S_{w c}}{1-S_{w c}-S_{o r}}\right)^{n_{w}} \\
& k_{r o}=k_{\text {rocw }}\left(\frac{1-S_{w}-S_{o r}}{1-S_{w c}-S_{o r}}\right)^{n_{o}}
\end{aligned}
$$

where $k_{r w r o}$ is the oil relative permeability at the irreducible water saturation $S_{w c}, k_{r w c w}$ is the water relative permeability at the residual oil saturation and $S_{o r}, n_{w}$, and $n_{o}$ are constant coefficients.

The polymer solution viscosity $\mu_{p}(\mathrm{mPa} \cdot \mathrm{s})$, the permeability reduction factor $R_{k}$, and the amount adsorbed per unit mass of the rock $C_{r p}(\mathrm{mg} / \mathrm{g})$ which depend on the polymer concentration $c_{p}$ are given by

$$
\begin{gathered}
\mu_{p}=\mu_{w}\left[1+\left(a p_{1} c_{p}+a p_{2} c_{p}^{2}+a p_{p}^{3}\right)\right], \\
R_{k}=1+\frac{\left(R_{k \max }-1\right) \cdot b_{r k} \cdot c_{p}}{1+b_{r k} \cdot c_{p}}, \\
C_{r p}=\frac{a c_{p}}{1+b c_{p}},
\end{gathered}
$$

where $\mu_{w}$ is the viscosity of the aqueous phase with no polymer (mPa.s), $a p_{1}, a p_{2}, a p_{3}$, $R_{k \text { max }}, b_{r k}$, and $a\left(\mathrm{~cm}^{3} / \mathrm{g}\right)$ and $b(\mathrm{~g} / \mathrm{L})$ are constant coefficients.

The fractional flow of water $f_{w}$ is given by,

$$
f_{w}=\frac{r_{w}}{r_{o}+r_{w}}
$$




\subsection{Constraint}

Since the negative and overhigh injection polymer concentrations are not allowed, the constraint in polymer flooding is expressed mathematically as

$$
0 \leq c_{\text {pin }} \leq c_{\max }
$$

where $c_{\max }$ is the maximum injection polymer concentration.

\subsection{Optimal Control Formulation}

The reservoir pressure $p$, the water saturation $S_{w}$ and the polymer concentration $c_{p}$ are the three state variables for the problem as formulated. The system state vector is denoted by

$$
\mathbf{u}(x, y, t)=\left[p, S_{w}, c_{p}\right]^{T}
$$

The control for the process is the polymer concentration of injected fluid

$$
v(x, y, t)=c_{\text {pin }}, \quad(x, y) \in L_{w}
$$

Then the OCP of DPS for polymer flooding has the general form,

$$
\begin{gathered}
\max _{v} J=\int_{0}^{t_{f}} \iint_{\Omega} F(\mathbf{u}, v) d \sigma d t, \\
\text { s.t. } \mathbf{f}\left(\dot{\mathbf{u}}, \mathbf{u}, \mathbf{u}_{x}, \mathbf{u}_{y}, \mathbf{u}_{x x}, \mathbf{u}_{y y}, v\right)=0, \quad(x, y, t) \in \Psi, \\
\mathbf{g}\left(\mathbf{u}, \mathbf{u}_{x}, \mathbf{u}_{y}, \mathbf{u}_{x x}, \mathbf{u}_{y y}\right)=0, \quad(x, y, t) \in \Gamma, \\
\mathbf{u}(x, y, 0)=\mathbf{u}^{0}(x, y), \quad(x, y) \in \Omega, \\
0 \leq v \leq v_{\max },
\end{gathered}
$$

where $\dot{\mathbf{u}}=\partial \mathbf{u} / \partial t, \mathbf{u}_{l}=\partial \mathbf{u} / \partial l, \mathbf{u}_{l l}=\partial^{2} \mathbf{u} / \partial l^{2}, l=x, y$, (2.19) denotes the performance index (2.3), (2.20) expresses the governing equations (2.4)-(2.6), (2.21) and (2.22) denote the boundary and initial conditions, respectively, and (2.23) denotes the injection polymer concentration constraint (2.16).

\section{Necessary Conditions of Optimal Control}

\subsection{Maximum Principle of DPS}

We desire to find a set of necessary conditions for the state vector, $\mathbf{u}$, and the control, $v$, to be extremals of the functional $J$ (2.19) subject to the PDEs (2.20) (2.22) and the constraint (2.23). A convenient way to cope with such an OCP of DPS (2.19) (2.23) is through the use 
of distributed adjoint variables. The first step is to form an augmented functional by adjoining the governing equations to the performance index $J$. We define the Hamiltonian as

$$
H=F+\lambda^{T} \mathbf{f}
$$

where $\mathcal{\lambda}(x, y, t)$ is the adjoint vector. Then the argument functional is given by,

$$
J_{A}=J+\int_{0}^{t_{f}} \iint_{\Omega} \Lambda^{T} \mathbf{f}\left(\dot{\mathbf{u}}, \mathbf{u}, \mathbf{u}_{x}, \mathbf{u}_{y}, \mathbf{u}_{x x}, \mathbf{u}_{y y}, v\right) d \sigma d t=\int_{0}^{t_{f}} \iint_{\Omega} H\left(\dot{\mathbf{u}}, \mathbf{u}, \mathbf{u}_{x}, \mathbf{u}_{y}, \mathbf{u}_{x x}, \mathbf{u}_{y y}, v\right) d \sigma d t
$$

Following the standard procedure of the calculus of variables, the increment of $J_{A}$, denoted by $\Delta J_{A}$, is formed by introducing variations $\delta \mathbf{u}, \delta \mathbf{u}_{x}, \delta \mathbf{u}_{y}, \delta \mathbf{u}_{x x}, \delta \mathbf{u}_{y y}, \delta \dot{\mathbf{u}}$, and $\delta v$ giving

$$
\begin{aligned}
\Delta J_{A}= & J_{A}\left(\mathbf{u}+\delta \mathbf{u}, \mathbf{u}_{x}+\delta \mathbf{u}_{x}, \mathbf{u}_{y}+\delta \mathbf{u}_{y}, \mathbf{u}_{x x}+\delta \mathbf{u}_{x x}, \mathbf{u}_{y y}+\delta \mathbf{u}_{y y}, \dot{\mathbf{u}}+\delta \dot{\mathbf{u}}, v+\delta v\right) \\
& -J_{A}\left(\mathbf{u}, \mathbf{u}_{x}, \mathbf{u}_{y}, \mathbf{u}_{x x}, \mathbf{u}_{y y}, \dot{\mathbf{u}}, v\right) .
\end{aligned}
$$

This formulation assumes that the final time, $t_{f}$, is fixed.

Expanding (3.3) in a Taylor series and retaining only the linear terms give the variation of the functional, $\delta J_{A}$,

$$
\begin{aligned}
& \delta J_{A}=\int_{0}^{t_{f}} \iint_{\Omega} {\left[\left(\frac{\partial H}{\partial \mathbf{u}}\right)^{T} \delta \mathbf{u}+\left(\frac{\partial H}{\partial \mathbf{u}_{x}}\right)^{T} \delta \mathbf{u}_{x}+\left(\frac{\partial H}{\partial \mathbf{u}_{x x}}\right)^{T} \delta \mathbf{u}_{x x}+\left(\frac{\partial H}{\partial \mathbf{u}_{y}}\right)^{T} \delta \mathbf{u}_{y}\right.} \\
&\left.+\left(\frac{\partial H}{\partial \mathbf{u}_{y y}}\right)^{T} \delta \mathbf{u}_{y y}+\left(\frac{\partial H}{\partial \dot{\mathbf{u}}}\right)^{T} \delta \dot{\mathbf{u}}+\left(\frac{\partial H}{\partial v}\right) \delta v\right] d \sigma d t
\end{aligned}
$$

Since the variations $\delta \mathbf{u}, \delta \mathbf{u}_{l}, \delta \mathbf{u}_{l l}(l=x, y)$, and $\delta \dot{\mathbf{u}}$ are not independent can be expressed in terms of the variations $\delta \mathbf{u}$ by integrating the following three terms by parts:

$$
\begin{aligned}
\iint_{\Omega}\left[\left(\frac{\partial H}{\partial \mathbf{u}_{l}}\right)^{T} \delta \mathbf{u}_{l}\right] d \sigma= & \iint_{\Omega} \frac{\partial}{\partial l}\left[\left(\frac{\partial H}{\partial \mathbf{u}_{l}}\right)^{T} \delta \mathbf{u}\right] d \sigma-\iint_{\Omega}\left[\frac{\partial}{\partial l}\left(\frac{\partial H}{\partial \mathbf{u}_{l}}\right)^{T} \delta \mathbf{u}\right] d \sigma, \\
\iint_{\Omega}\left[\left(\frac{\partial H}{\partial \mathbf{u}_{l l}}\right)^{T} \delta \mathbf{u}_{l l}\right] d \sigma= & \iint_{\Omega}\left[\frac{\partial^{2}}{\partial l^{2}}\left(\frac{\partial H}{\partial \mathbf{u}_{l l}}\right)\right]^{T} \delta \mathbf{u} d \sigma \\
& +\iint_{\Omega} \frac{\partial}{\partial l}\left[\left(\frac{\partial H}{\partial \mathbf{u}_{l l}}\right)^{T} \delta \mathbf{u}_{l}-\frac{\partial}{\partial l}\left(\frac{\partial H}{\partial \mathbf{u}_{l l}}\right)^{T} \delta \mathbf{u}\right] d \sigma, \\
\int_{0}^{t_{f}}\left(\frac{\partial H}{\partial \dot{\mathbf{u}}}\right)^{T} \delta \dot{\mathbf{u}}= & {\left.\left[\left(\frac{\partial H}{\partial \dot{\mathbf{u}}}\right)^{T} \delta \mathbf{u}\right]\right|_{0} ^{t_{f}}-\int_{0}^{t_{f}} \frac{\partial}{\partial t}\left(\frac{\partial H}{\partial \dot{\mathbf{u}}}\right)^{T} \delta \mathbf{u} d t . }
\end{aligned}
$$


Using the Green's formula in (3.5) and (3.6), we obtain

$$
\begin{aligned}
& \iint_{\Omega_{l}=x, y} \sum_{l}\left[\left(\frac{\partial H}{\partial \mathbf{u}_{l}}\right)^{T} \delta \mathbf{u}_{l}+\left(\frac{\partial H}{\partial \mathbf{u}_{l l}}\right)^{T} \delta \mathbf{u}_{l l}\right] d \sigma \\
&=\iint_{\Omega_{l}=x, y} \sum_{l}\left[-\frac{\partial}{\partial l}\left(\frac{\partial H}{\partial \mathbf{u}_{l}}\right)+\frac{\partial^{2}}{\partial l^{2}}\left(\frac{\partial H}{\partial \mathbf{u}_{l l}}\right)\right]^{T} \delta \mathbf{u} d \sigma \\
&+\oint_{\partial \Omega}\left\{\left[\left(\frac{\partial H}{\partial \mathbf{u}_{x}}-\frac{\partial}{\partial x} \frac{\partial H}{\partial \mathbf{u}_{x x}}\right)^{T} \delta \mathbf{u}+\left(\frac{\partial H}{\partial \mathbf{u}_{x x}}\right)^{T} \delta \mathbf{u}_{x}\right] d y\right. \\
&\left.-\left[\left(\frac{\partial H}{\partial \mathbf{u}_{y}}-\frac{\partial}{\partial y} \frac{\partial H}{\partial \mathbf{u}_{y y}}\right)^{T} \delta \mathbf{u}+\left(\frac{\partial H}{\partial \mathbf{u}_{y y}}\right)^{T} \delta \mathbf{u}_{y}\right] d x\right\}
\end{aligned}
$$

By substituting the above equations (3.7) and (3.8) into (3.4), the first variation $\delta J_{A}$ is expressed as

$$
\begin{aligned}
\delta J_{A}= & \int_{t_{0}}^{t_{f}} \iint_{\Omega}\left(\frac{\partial H}{\partial \mathbf{u}}-\frac{\partial}{\partial x} \frac{\partial H}{\partial \mathbf{u}_{x}}-\frac{\partial}{\partial y} \frac{\partial H}{\partial \mathbf{u}_{y}}+\frac{\partial^{2}}{\partial x^{2}} \frac{\partial H}{\partial \mathbf{u}_{x x}}+\frac{\partial^{2}}{\partial y^{2}} \frac{\partial H}{\partial \mathbf{u}_{y y}}-\frac{\partial}{\partial t} \frac{\partial H}{\partial \dot{\mathbf{u}}}\right)^{T} \delta \mathbf{u} d \sigma d t \\
& +\int_{t_{0}}^{t_{f}} \oint_{\partial \Omega}\left\{\left[\left(\frac{\partial H}{\partial \mathbf{u}_{x}}-\frac{\partial}{\partial x} \frac{\partial H}{\partial \mathbf{u}_{x x}}\right)^{T} \delta \mathbf{u}+\left(\frac{\partial H}{\partial \mathbf{u}_{x x}}\right)^{T} \delta \mathbf{u}_{x}\right] d y\right. \\
& \left.-\left[\left(\frac{\partial H}{\partial \mathbf{u}_{y}}-\frac{\partial}{\partial y} \frac{\partial H}{\partial \mathbf{u}_{y y}}\right)^{T} \delta \mathbf{u}+\left(\frac{\partial H}{\partial \mathbf{u}_{y y}}\right)^{T} \delta \mathbf{u}_{y}\right] d x\right\} d t \\
& +\left.\iint_{\Omega}\left[\left(\frac{\partial H}{\partial \dot{\mathbf{u}}}\right)^{T} \delta \mathbf{u}\right]\right|_{0} ^{t_{f}} d \sigma+\int_{t_{0}}^{t_{f}} \iint_{\Omega}\left(\frac{\partial H}{\partial v}\right) \delta v d \sigma d t .
\end{aligned}
$$

When the state and control regions are not bounded, the variation of the functional must vanish at an extremal (the fundamental theorem of the calculus of variations). When the control region is constrained by a boundary, then the necessary condition for optimality is to maximize the performance index $J_{A}$ with respect to the control $v$. This means that the variation $\delta J_{A}$ is

$$
\delta J_{A}\left(v^{*}, \delta v\right) \geq 0,
$$

where $v^{*}$ denotes the optimal control. Equation (3.10) is the weak minimum principle of Pontryagin. The necessary conditions for these two cases are the same except for the term involving the variation of the control, $\delta v$. For polymer flooding problem there are higher and lower bounds on the control variable $v$ given as (2.16).

The following necessary conditions for optimality are obtained when we apply Pontryagin's maximum principle. 


\section{(1) Adjoint Equations}

Since the variation $\delta \mathbf{u}$ is free and not zero, the coefficient terms involving the $\delta \mathbf{u}$ variation in the first term of (3.9) are set to zero. This results in the adjoint equations as given by

$$
\frac{\partial H}{\partial \mathbf{u}}-\sum_{l=x, y}\left(\frac{\partial}{\partial l} \frac{\partial H}{\partial \mathbf{u}_{l}}+\frac{\partial^{2}}{\partial l^{2}} \frac{\partial H}{\partial \mathbf{u}_{l l}}\right)-\frac{\partial}{\partial t} \frac{\partial H}{\partial \dot{\mathbf{u}}}=0 .
$$

Substitute the Hamiltonian (3.1) into (3.11) and the adjoint equations become

$$
\begin{aligned}
\frac{\partial F}{\partial \mathbf{u}} & +\left(\frac{\partial \mathbf{f}}{\partial \mathbf{u}}-\frac{\partial}{\partial t} \frac{\partial \mathbf{f}}{\partial \dot{\mathbf{u}}}\right)^{T} \lambda \\
& +\sum_{l=x, y}\left[\left(\frac{\partial^{2}}{\partial l^{2}} \frac{\partial \mathbf{f}}{\partial \mathbf{u}_{l l}}-\frac{\partial}{\partial l} \frac{\partial \mathbf{f}}{\partial \mathbf{u}_{l}}\right)^{T} \mathcal{\lambda}+\left(2 \frac{\partial}{\partial l} \frac{\partial \mathbf{f}}{\partial \mathbf{u}_{l l}}-\frac{\partial \mathbf{f}}{\partial \mathbf{u}_{l}}\right)^{T} \frac{\partial \boldsymbol{l}}{\partial l}+\left(\frac{\partial \mathbf{f}}{\partial \mathbf{u}_{l l}}\right)^{T} \frac{\partial^{2} \boldsymbol{\jmath}}{\partial l^{2}}\right] \\
& -\left(\frac{\partial \mathbf{f}}{\partial \dot{\mathbf{u}}}\right)^{T} \frac{\partial \boldsymbol{l}}{\partial t}=0 .
\end{aligned}
$$

Equation (3.12) is a set of PDEs with nonconstant coefficients.

\section{(2) Transversality Boundary Conditions}

The adjoint boundary conditions are obtained from the second term of (3.9):

$$
\left.\left[\left(\frac{\partial H}{\partial \mathbf{u}_{l}}-\frac{\partial}{\partial l} \frac{\partial H}{\partial \mathbf{u}_{l l}}\right)^{T} \delta \mathbf{u}+\left(\frac{\partial H}{\partial \mathbf{u}_{l l}}\right)^{T} \delta \mathbf{u}_{l}\right]\right|_{\partial \Omega}=0, \quad l=x, y .
$$

\section{(3) Transversality Terminal Conditions}

Since the initial state is specified, the variation $\left.\delta \mathbf{u}\right|_{t=0}$ of (3.9) is zero. However, the final state is not specified; therefore, the variation $\left.\delta \mathbf{u}\right|_{t=t_{f}}$ is free and nonzero. This means that the following relation must be zero:

$$
\frac{\partial H}{\partial \dot{\mathbf{u}}}=\left(\frac{\partial f}{\partial \dot{\mathbf{u}}}\right)^{T} \lambda=0, \text { at } t=t_{f}
$$

\section{(4) Optimal Control}

With all the previous terms vanishing, the variation of the functional $\delta J_{A}$ becomes

$$
\delta J_{A}=\int_{0}^{t_{f}} \iint_{\Omega}\left(\frac{\partial H}{\partial v}\right) \delta v d \sigma d t
$$

This equation expresses the direct influence of variation $\delta v$ on $\delta J_{A}$. A necessary condition for the optimality of $v^{*}$ is that $\delta J_{A} \geq 0$ for all possible small variations, $\delta v$. Since there are lower 
and higher bounds on the control $v(2.9)$, we use the weak maximum principle to assert the following necessary conditions for optimality:

$$
\frac{\partial H}{\partial v}=0 \quad \text { for } 0 \leq v^{*} \leq v_{\max }
$$

when the control vector is unconstrained. Because the variation $\delta v$ can only be negative along the lower bound, we have

$$
\frac{\partial H}{\partial v} \leq 0 \quad \text { for } v^{*}=0
$$

And because the variation $\delta v$ can only be positive along the higher bound, we have

$$
\frac{\partial H}{\partial v} \geq 0 \quad \text { for } v^{*}=v_{\max }
$$

\subsection{Necessary Conditions of OCP for Polymer Flooding}

Let $\lambda(x, y, t)=\left(\lambda_{1}, \lambda_{2}, \lambda_{3}\right)^{T}$ denote the adjoint vector of OCP for polymer flooding. Applying the theory developed in Section 3.1 and substituting the governing equations (2.4)-(2.6) into (3.12), the adjoint equations, given by (3.12), reduce for the polymer flooding problem under consideration as given in,

$$
\begin{aligned}
& \sum_{l=x, y}\{ \frac{\partial}{\partial l}\left(k_{p} r_{o} \frac{\partial \lambda_{1}}{\partial l}\right)+\frac{\partial}{\partial l}\left(k_{p} r_{w} \frac{\partial \lambda_{2}}{\partial l}\right)+\frac{\partial}{\partial l}\left(k_{p} r_{c} \frac{\partial \lambda_{3}}{\partial l}\right) \\
&\left.-\left[k_{p} \frac{\partial r_{o}}{\partial p} \frac{\partial p}{\partial l} \frac{\partial \lambda_{1}}{\partial l}+k_{d} \frac{\partial r_{w}}{\partial p} \frac{\partial p}{\partial l} \frac{\partial \lambda_{2}}{\partial l}+\left(k_{p} \frac{\partial r_{c}}{\partial p} \frac{\partial p}{\partial l}+k_{d} \frac{\partial r_{d}}{\partial p} \frac{\partial c_{p}}{\partial l}\right) \frac{\partial \lambda_{3}}{\partial l}\right]\right\} \\
&-q_{\text {out }}\left(\xi_{o} \frac{\partial f_{w}}{\partial p}-\frac{\partial f_{w}}{\partial p} \lambda_{1}+\frac{\partial f_{w}}{\partial p} \lambda_{2}+c_{p} \frac{\partial f_{w}}{\partial p} \lambda_{3}\right) \\
&+\frac{\partial a_{o}}{\partial p} \frac{\partial \lambda_{1}}{\partial t}+\frac{\partial a_{w}}{\partial p} \frac{\partial \lambda_{2}}{\partial t}+\frac{\partial a_{c}}{\partial p} \frac{\partial \lambda_{3}}{\partial t}=0, \quad(x, y, t) \in \Psi, \\
& \sum_{l=x, y}\left[-k_{p} \frac{\partial p}{\partial l}\left(\frac{\partial r_{o}}{\partial S_{w}} \frac{\partial \lambda_{1}}{\partial l}+\frac{\partial r_{w}}{\partial S_{w}} \frac{\partial \lambda_{2}}{\partial l}+\frac{\partial r_{c}}{\partial S_{w}} \frac{\partial \lambda_{3}}{\partial l}\right)-k_{d} \frac{\partial r_{d}}{\partial S_{w}} \frac{\partial c_{p}}{\partial l} \frac{\partial \lambda_{3}}{\partial l}\right] \\
&-q_{\text {out }}\left(\xi_{o} \frac{\partial f_{w}}{\partial S_{w}}-\frac{\partial f_{w}}{\partial S_{w}} \lambda_{1}+\frac{\partial f_{w}}{\partial S_{w}} \lambda_{2}+c_{p} \frac{\partial f_{w}}{\partial S_{w}} \lambda_{3}\right)+\frac{\partial a_{o}}{\partial S_{w}} \frac{\partial \lambda_{1}}{\partial t}+\frac{\partial a_{w}}{\partial S_{w}} \frac{\partial \lambda_{2}}{\partial t} \\
&+\frac{\partial a_{c}}{\partial S_{w}} \frac{\partial \lambda_{3}}{\partial t}=0, \quad(x, y, t) \in \Psi,
\end{aligned}
$$




$$
\begin{aligned}
\sum_{l=x, y}[ & \left.\frac{\partial}{\partial l}\left(k_{d} r_{d} \frac{\partial \lambda_{3}}{\partial l}\right)-k_{p} \frac{\partial p}{\partial l}\left(\frac{\partial r_{w}}{\partial c_{p}} \frac{\partial \lambda_{2}}{\partial l}+\frac{\partial r_{c}}{\partial c_{p}} \frac{\partial \lambda_{3}}{\partial l}\right)\right] \\
- & q_{\text {out }}\left[\xi_{o} \frac{\partial f_{w}}{\partial c_{p}}-\frac{\partial f_{w}}{\partial c_{p}} \lambda_{1}+\frac{\partial f_{w}}{\partial c_{p}} \lambda_{2}+\left(c_{p} \frac{\partial f_{w}}{\partial c_{p}}+f_{w}\right) \lambda_{3}\right] \\
+ & \frac{\partial a_{c}}{\partial c_{p}} \frac{\partial \lambda_{3}}{\partial t}=0, \quad(x, y, t) \in \Psi .
\end{aligned}
$$

The boundary conditions (2.7) of the DPS result in $\left.(\partial \mathbf{u} / \partial l)\right|_{\partial \Omega}=0$ and $\left.\delta \mathbf{u}_{l}\right|_{\partial \Omega}=0, l=$ $x, y$, in (3.13). The coefficients of the arbitrary variation $\left.\delta \mathbf{u}_{l}\right|_{\partial \Omega}$ terms must be zero and yield the boundary conditions for the adjoint equations as given by

$$
\frac{\partial H}{\partial \mathbf{u}_{l}}-\frac{\partial}{\partial l} \frac{\partial H}{\partial \mathbf{u}_{l l}}=0, \quad l=x, y
$$

By substituting the governing equations (2.4)-(2.6) into (3.20), the boundary conditions of adjoint equations for the polymer flooding OCP are expressed as

$$
\left.\left(r_{o} \frac{\partial \lambda_{1}}{\partial l}+r_{w} \frac{\partial \lambda_{2}}{\partial l}\right)\right|_{\partial \Omega}=0,\left.\quad \frac{\partial \lambda_{3}}{\partial l}\right|_{\partial \Omega}=0, \quad l=x, y,(x, y, t) \in \Gamma .
$$

The following transversality terminal conditions at $t=t_{f}$ are obtained by substituting the governing equations (2.4)-(2.6) into (3.14):

$$
\begin{gathered}
-\frac{\partial a_{o}}{\partial p} \lambda_{1}-\frac{\partial a_{w}}{\partial p} \lambda_{2}-\frac{\partial a_{c}}{\partial p} \lambda_{3}=0 \\
-\frac{\partial a_{o}}{\partial S_{w}} \lambda_{1}-\frac{\partial a_{w}}{\partial S_{w}} \lambda_{2}-\frac{\partial a_{c}}{\partial S_{w}} \lambda_{3}=0, \quad-\frac{\partial a_{c}}{\partial c_{p}} \lambda_{3}=0
\end{gathered}
$$

Since the coefficient terms involving the adjoint variables in (3.22) are not zero, the terminal conditions of adjoint equations in the OCP of polymer flooding can be simplified to

$$
\lambda_{1}\left(x, y, t_{f}\right)=0, \quad \lambda_{2}\left(x, y, t_{f}\right)=0, \quad \lambda_{3}\left(x, y, t_{f}\right)=0, \quad(x, y) \in \Omega
$$

Equation (3.23) shows that the adjoint variables are known at the final time $t_{f}$. Since the state variables are known at the initial time and the adjoint variables are known at the final time, the OCP is a split two-point boundary-value problem.

The variation of the performance index, $J$, reduces to the following simplified functional of the control variation:

$$
\delta J_{A}=\int_{0}^{t_{f}} \iint_{\Omega} q_{\text {in }}\left(\xi_{p}+\lambda_{3}\right) \delta v d \sigma d t
$$


From the results of (3.16)-(3.18), the necessary condition for optimality of polymer flooding problem is

$$
q_{\text {in }}\left(\xi_{p}+\lambda_{3}\right) \begin{cases}=0, & \text { for } 0 \leq v^{*} \leq v_{\max } \\ \leq 0, & \text { for } v^{*}=0 \\ \geq 0, & \text { for } v^{*}=v_{\max }\end{cases}
$$

\section{Numerical Solution}

We propose an iterative numerical technique for determining the optimal injection strategies of polymer flooding. The computational procedure is based on adjusting estimates of control function $v$ to improve the value of the objective functional. For a control to be optimal, the necessary condition given by (3.25) must be satisfied. If the control $v$ is not optimal, then a correction $\delta v$ is determined so that the functional is made lager, that is, $\delta J_{A}>0$. If $\delta v$ is selected as

$$
\delta v=w q_{\text {in }}\left(\xi_{p}+\lambda_{3}\right)
$$

where $w$ is an arbitrary positive weighting factor, the functional variation becomes

$$
\delta J_{A}=\int_{0}^{t_{f}} \iint_{\Omega} w\left[q_{\text {in }}\left(\xi_{p}+\lambda_{3}\right)\right]^{2} d \sigma d t \geq 0
$$

Thus, choosing $\delta v$ in the gradient direction ensures a local improvement in the objective functional, $J_{A}$. At the higher and lower bounds on $v$, we must make the appropriate weighting terms equal to zero to avoid leaving the allowable region.

The computational algorithm of control iteration based on gradient direction is as follows.

\section{(1) Initialization}

Make an initial guess for the control function, $v(x, y, t),(x, y) \in L_{w}, t \in\left[0, t_{f}\right]$.

\section{(2) Resolution of the State Equations}

Using stored current value of $v(x, y, t),(x, y) \in L_{w}$, integrate the governing equations forward in time with known initial state conditions. We use the finite difference method of a full implicit scheme for the PDEs as discussed in [21, 22]. The profit functional is evaluated, and the coefficients involved in the adjoint equations which are function of the state solution are computed and stored.

\section{(3) Resolution of the Adjoint Equations}

Using the stored coefficients, integrate the adjoint equations numerically backward in time with known final time adjoint conditions by (3.23). Compute and store $\delta v$ as defined by (4.1). 


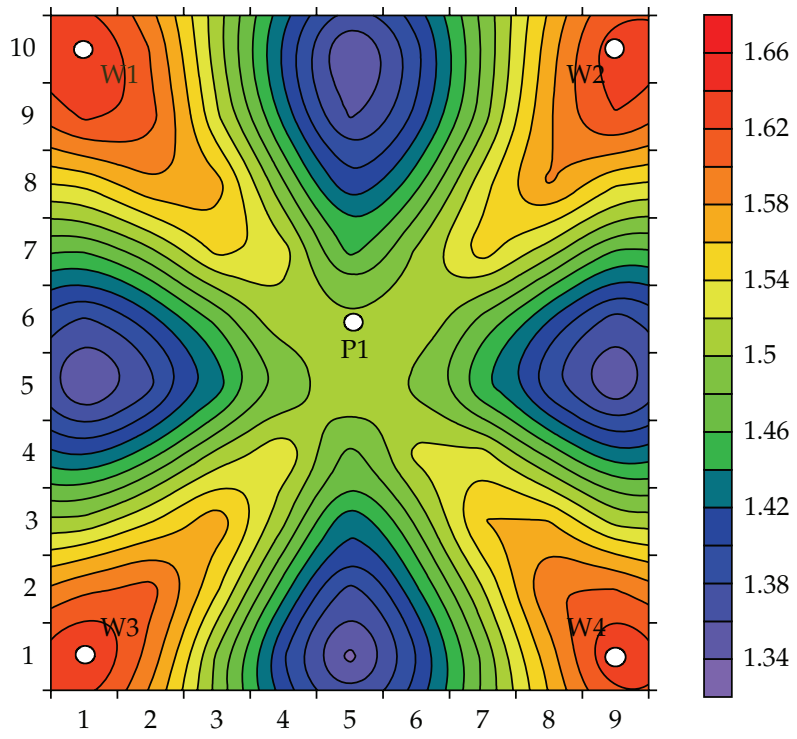

Figure 1: Permeability $\left(\mu \mathrm{m}^{2}\right)$ distribution.

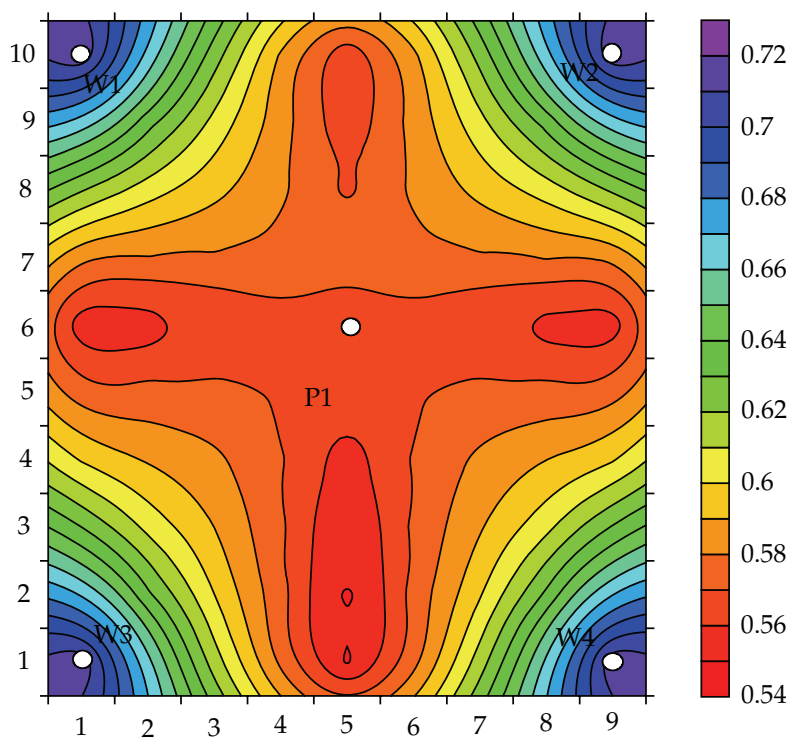

Figure 2: Initial water saturation contour map.

\section{(4) Computation of the New Control}

Using the evaluated $\delta v$, an improved function is computed as

$$
v(x, y, t)^{\text {new }}=v(x, y, t)^{\text {old }}+\delta v(x, y, t), \quad(x, y) \in L_{w},
$$




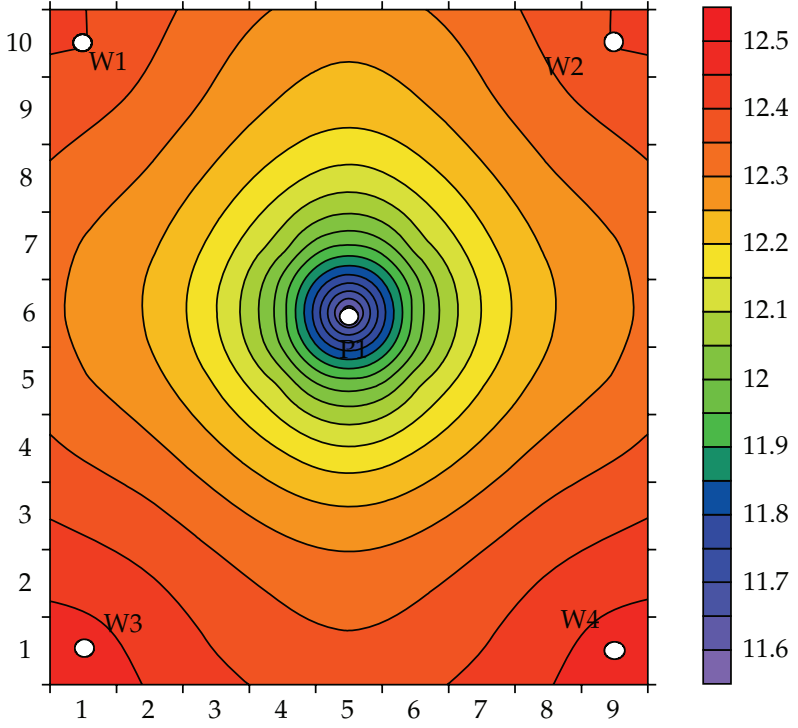

Figure 3: Initial reservoir pressure ( $\mathrm{MPa})$ contour map.

Table 1: Parameters of reservoir description used in the example.

\begin{tabular}{lc}
\hline Parameters & Values \\
\hline Number of production well, $N_{p}$ & 1 \\
Number of injection wells, $N_{w}$ & 4 \\
Thickness of the reservoir bed, $h(\mathrm{~m})$ & 5 \\
Reference pressure, $p_{r}(\mathrm{MPa})$ & 12 \\
Porosity under the condition of the reference pressure, $\phi_{r}$ & 0.31 \\
Rock density, $\rho_{r}\left(\mathrm{~kg} / \mathrm{m}^{3}\right)$ & 2000 \\
Rock compressibility factor, $C_{R}(1 / \mathrm{MPa})$ & $9.38 \times 10^{-6}$ \\
Irreducible water saturation, $S_{o r}$ & 0.25 \\
Residual oil saturation, $S_{w c}$ & 0.22 \\
Oil relative permeability at the irreducible water saturation, $k_{r w r o}$ & 0.5228 \\
Water relative permeability at the residual oil saturation, $k_{\text {rocw }}$ & 0.9 \\
Index of oil relative permeability curve, $n_{o}$ & 4.287 \\
Index of water relative permeability curve, $n_{w}$ & 2.3447 \\
\hline
\end{tabular}

where $0 \leq v^{\text {new }} \leq v_{\max }$. A single variable search strategy can be used to find the value of the positive weighting factor $w$ which maximizes the improvement in the performance functional using (4.3).

\section{(5) Termination}

The optimization algorithm is stopped when the variation $\delta v$ is too small to effectively change the performance measure, that is, when

$$
\left|J^{\text {new }}-J^{\text {old }}\right|<\varepsilon
$$

where $\varepsilon$ is a small positive number. 
Table 2: Fluid data used in the example.

\begin{tabular}{lc}
\hline Parameters & Values \\
\hline Oil viscosity, $\mu_{o}$ (mPa·s) & 50 \\
Compressibility factors of oil, $C_{o}(1 / \mathrm{MPa})$ & $5 \times 10^{-6}$ \\
Oil volume factor under the condition of the reference pressure, $B_{\text {or }}$ & 1 \\
Aqueous phase viscosity with no polymer, $\mu_{w}(\mathrm{mPa} \cdot \mathrm{s})$ & 0.458 \\
Compressibility factors of water, $C_{w}(1 / \mathrm{MPa})$ & $4.6 \times 10^{-6}$ \\
Water volume factor under the condition of the reference pressure, $B_{w r}$ & 1 \\
Polymer absorption parameter, $a\left(\mathrm{~g} / \mathrm{cm}^{3}\right)$ & 0.03 \\
Polymer absorption parameter, $b\left(\mathrm{~g} / \mathrm{cm}^{3}\right)$ & 3.8 \\
Diffusion coefficient, $D\left(\mathrm{~m}^{2} / \mathrm{s}\right)$ & $1 \times 10^{-5}$ \\
Effective pore volume coefficient, $f_{a}$ & 1 \\
Permeability reduction parameter, $R_{k \max }$ & 1.15 \\
Permeability reduction parameter, $b_{r k}$ & 1.2 \\
Viscosity parameter, $a p_{1}$ & 15.426 \\
Viscosity parameter, $a p_{2}$ & 0.4228 \\
Viscosity parameter, $a p_{3}$ & 0.2749 \\
\hline
\end{tabular}

\section{Case Study}

In this section we present a numerical example of optimal control for polymer flooding done with the proposed iterative gradient method.

The two-phase flow of oil and water in a heterogeneous two-dimensional reservoir is considered. The reservoir covers an area of $421.02 \times 443.8 \mathrm{~m}^{2}$ and has a thickness of $5 \mathrm{~m}$ and is discretized into $90(9 \times 10 \times 1)$ grid blocks. The production model is a five-spot pattern, with one production well P1 located at the center of the reservoir $(5,6)$ and four injection wells W1 W4 placed at the four corners $(1,10),(9,10),(1,1)$, and $(9,1)$ as shown in the permeability distribution map of Figure 1. Polymer is injected when the fractional flow of water for the production well comes to $97 \%$ after water flooding. The time domain of polymer injection is $0 \sim 1440$ days and the polymer flooding project life is $t_{f}=5500$ (days). Figures 2 and 3 show the contour maps of the initial water saturation $S_{w}^{0}$ and the initial reservoir pressure $p^{0}$, respectively. The initial polymer concentration is $c_{p}^{0}=0(\mathrm{~g} / \mathrm{L})$. In the performance index calculation, we use the price of oil $\xi_{o}=0.0503\left(10^{4} \$ / \mathrm{m}^{3}\right)(80(\$ / \mathrm{bbl}))$, and the cost of polymer $\xi_{p}=2.5 \times 10^{-4}\left(10^{4} \$ / \mathrm{kg}\right)$. The fluid velocity of production well is $q_{\text {out }}=7.225 \times$ $10^{-3}(\mathrm{~m} /$ day $)$, and the fluid velocity of every injection well is $q_{\text {in }}=2.89 \times 10^{-2}(\mathrm{~m} /$ day $)$. The PDEs are solved by full implicit finite difference method with step size 10 days. For the constraint (29), the maximum injection polymer concentration is $c_{\max }=2.2(\mathrm{~g} / \mathrm{L})$. The parameters of the reservoir description and the fluid data are shown in Tables 1 and 2, respectively.

The polymer injection strategies obtained by the conventional engineering judgment method (trial and error) are the same $1.8(\mathrm{~g} / \mathrm{L})$ for all injection wells. The performance index is $J=\$ 1.592 \times 10^{7}$ with oil production $32429 \mathrm{~m}^{3}$ and polymer injection $155520 \mathrm{~kg}$. For comparison, the results obtained by engineering judgment method are considered as the initial control strategies of the proposed iterative gradient method. A backtracking search strategy [23] is used to find the appropriate weighting term $w$ and the stopping criterion is chosen as $\varepsilon=1 \times 10^{-5}$. By using the proposed algorithm, we obtain a cumulative oil of $33045 \mathrm{~m}^{3}$ and a cumulative polymer of $151618 \mathrm{~kg}$ yielding the profit of $J^{*}=\$ 1.624 \times 10^{7}$ 


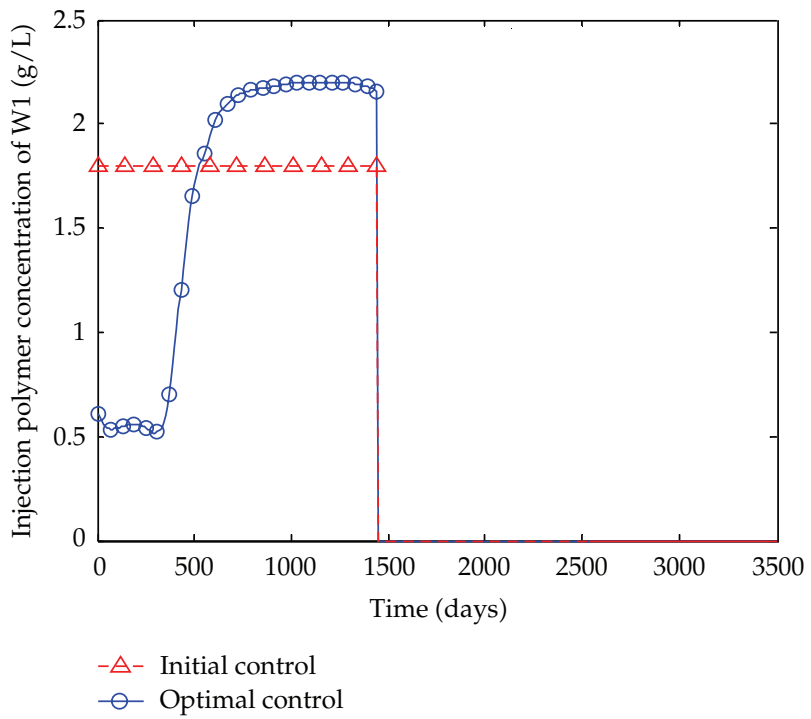

Figure 4: Injection polymer concentration of well W1.

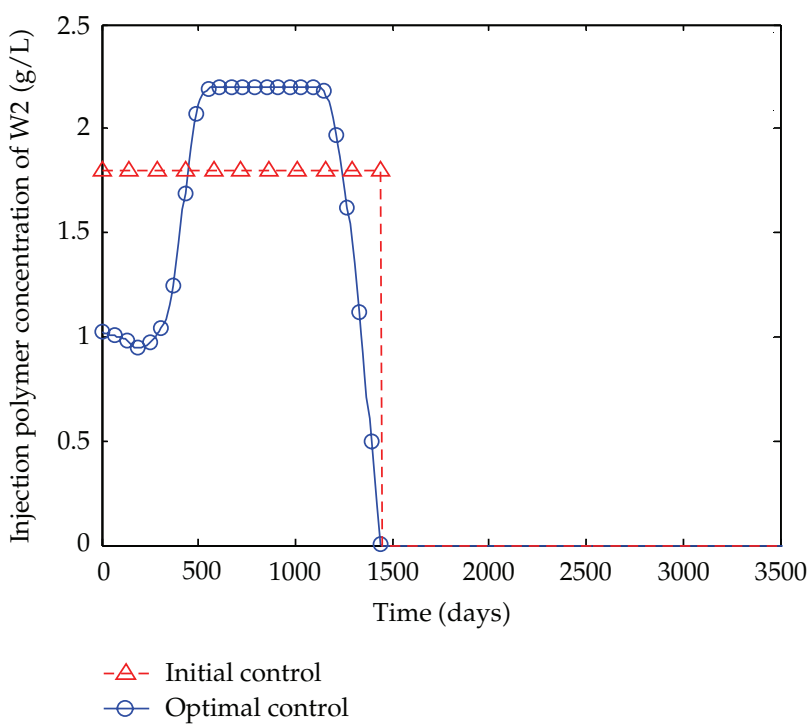

Figure 5: Injection polymer concentration of well W2.

over the polymer flooding project life of the reservoir. The results show an increase in performance index of $\$ 3.2 \times 10^{5}$. Figures 8 and 9 show the fractional flow of water in production well and the cumulative oil production curves of the two methods, respectively. It is obvious that the fractional flow of water obtained by iterative gradient method is lower than that by engineering judgment. Therefore, with the less cumulative polymer injection, the proposed method gets more oil production and higher recovery ratio. Figure 4 to Figure 7 show the optimal control policies of the injection wells W1 W4. As a result, the optimal injection polymer concentration profiles of W1 and W2 are significantly different 


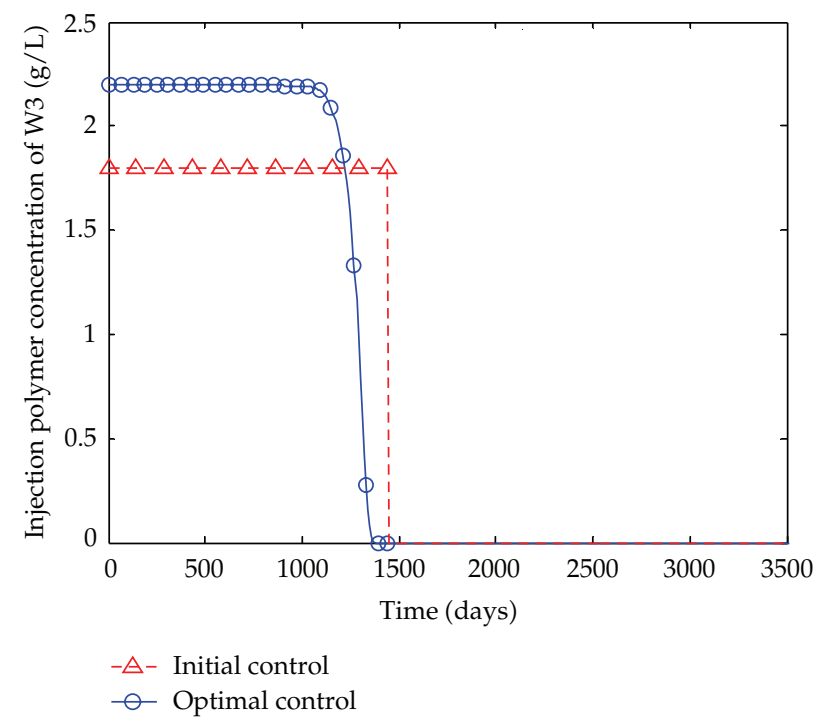

Figure 6: Injection polymer concentration of well W3.

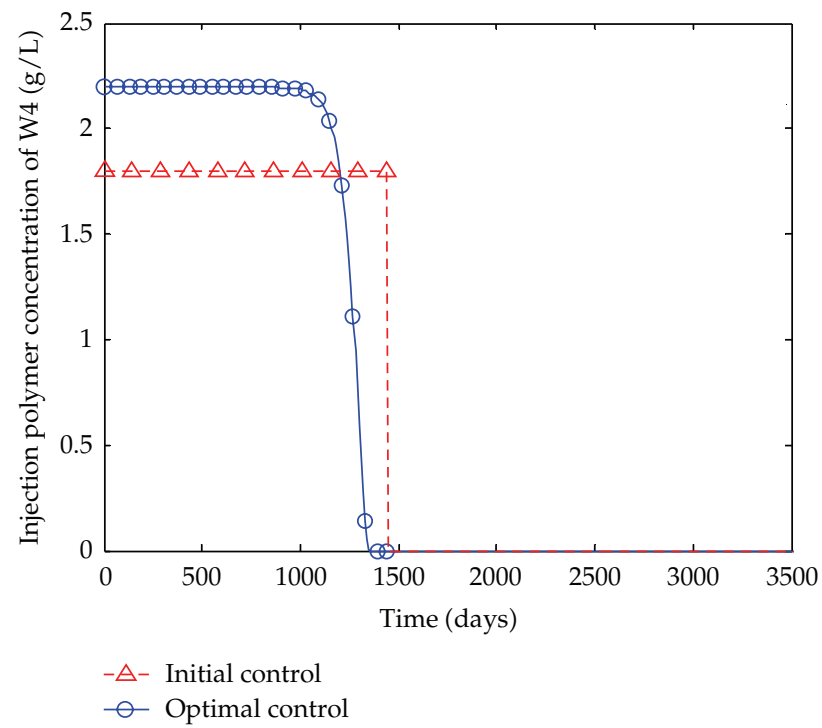

Figure 7: Injection polymer concentration of well W4.

from those of W3, W4. It is mainly due to the differences of the well positions and the distance to the production well, as well as the reservoir heterogeneity and the uniform initial water saturation distribution.

\section{Conclusion}

In this work, a new optimal control model of DPS is established for the dynamic injection strategies making of polymer flooding. Necessary conditions of this OCP are obtained by 


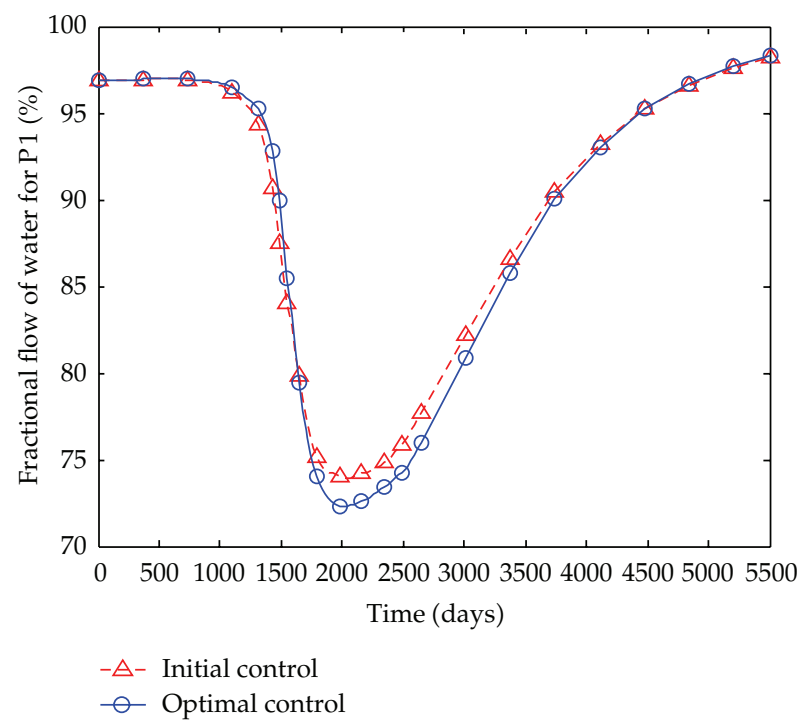

Figure 8: Fractional flow of water for the production well P1.

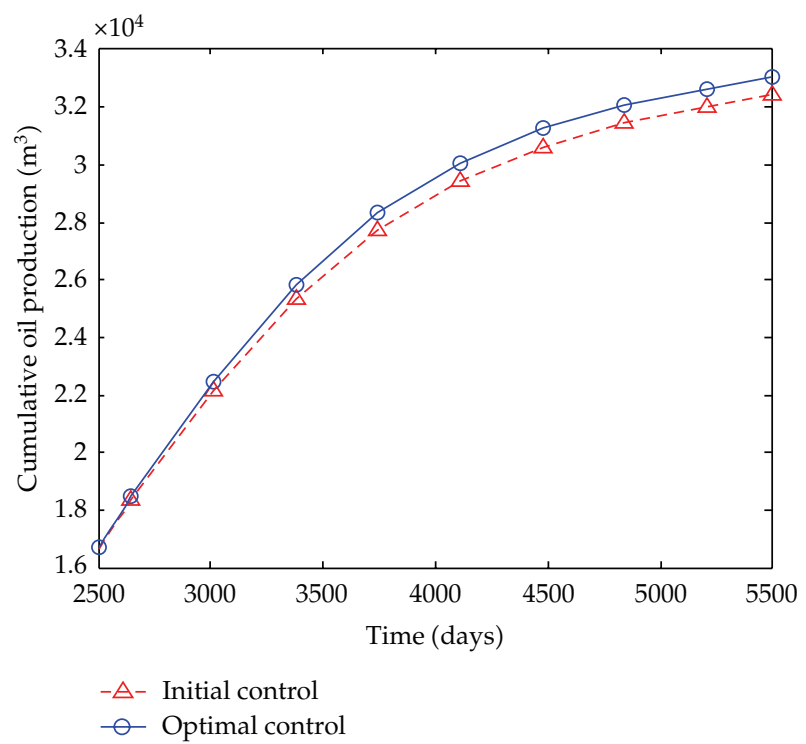

Figure 9: Cumulative oil production.

using the calculus of variations and Pontryagin's weak maximum principle. An iterative computational algorithm is proposed for the determination of optimal injection strategies. The optimal control model of polymer flooding and the proposed method are used for a reservoir example and the optimum injection concentration profiles for each well are offered. The results show that the profit is enhanced by the proposed method. Meanwhile, more oil production and higher recovery ratio are obtained. And the injection strategies chosen by engineering judgment are same for all the wells, whereas the optimal control policies by the 
proposed method are different from each other as a result of the reservoir heterogeneity and the uniform initial conditions.

In conclusion, given the properties of an oil reservoir and the properties of a polymer solution, optimal polymer flooding injection strategies to maximize profit can be designed by using distributed-parameter control theory. The approach used is a powerful tool that can aid significantly in the development of operational strategies for EOR processes.

\section{Acknowledgments}

This work was supported by the Natural Science Foundation of China under Grant 60974039, the Natural Science Foundation of Shandong Province of China under Grant ZR2011FM002, the Fundamental Research Funds for the Central Universities under Grant 27R1105018A, and the Postgraduate Innovation Funds of China University of Petroleum.

\section{References}

[1] Y. Qing, D. Caili, W. Yefei, T. Engao, Y. Guang, and Z. Fulin, “A study on mass concentration determination and property variations of produced polyacrylamide in polymer flooding," Petroleum Science and Technology, vol. 29, no. 3, pp. 227-235, 2011.

[2] B. K. Maitin, "Performance analysis of several polyacrylamide floods in North German oil fields," in Proceedings of the SPE/DOE Enhanced Oil Recovery Symposium, pp. 159-165, 1992.

[3] M. A. de Melo, C. R. C. de Holleben, I. P. G. da Silva et al., "Evaluation of polymer injection projects in Brazil," in Proceedings of the SPE Latin American and Caribbean Petroleum Engineering Conference, pp. 1$17,2005$.

[4] Q. Yu, H. Jiang, and C. Zhao, "Study of interfacial tension between oil and surfactant polymer flooding," Petroleum Science and Technology, vol. 28, no. 18, pp. 1846-1854, 2010.

[5] H. Jiang, Q. Yu, and Z. Yi, "The influence of the combination of polymer and polymer-surfactant flooding on recovery," Petroleum Science and Technology, vol. 29, no. 5, pp. 514-521, 2011.

[6] W. F. Ramirez, Z. Fathi, and J. L. Cagnol, "Optimal injection policies for enhanced oil recovery: part 1 theory and computational strategies," Society of Petroleum Engineers Journal, vol. 24, no. 3, pp. 328-332, 1984.

[7] Z. Fathi and W. F. Ramirez, "Use of optimal control theory for computing optimal injection policies for enhanced oil recovery," Automatica, vol. 22, no. 1, pp. 33-42, 1986.

[8] W. Liu, W. F. Ramirez, and Y. F. Qi, "Optimal control of steamflooding," SPE Advanced Technology Series, vol. 1, no. 2, pp. 73-82, 1993.

[9] J. Ye, Y. Qi, and Y. Fang, "Application of optimal control theory to making gas-cycling decision of condensate reservoir," Chinese Journal of Computational Physics, vol. 15, no. 1, pp. 71-76, 1998.

[10] P. Daripa, J. Glimm, B. Lindquist, and O. McBryan, "Polymer floods: a case study of nonlinear wave analysis and of instability control in tertiary oil recovery," SIAM Journal on Applied Mathematics, vol. 48, no. 2, pp. 353-373, 1988.

[11] P. Daripa and G. Paşa, "An optimal viscosity profile in enhanced oil recovery by polymer flooding," International Journal of Engineering Science, vol. 42, no. 19-20, pp. 2029-2039, 2004.

[12] P. Daripa and G. Paşa, "Stabilizing effect of diffusion in enhanced oil recovery and three-layer HeleShaw flows with viscosity gradient," Transport in Porous Media, vol. 70, no. 1, pp. 11-23, 2007.

[13] P. Daripa and G. Pasa, "On diffusive slowdown in three-layer Hele-Shaw flows," Quarterly of Applied Mathematics, vol. 68, no. 3, pp. 591-606, 2010.

[14] P. Daripa, "Studies on stability in three-layer Hele-Shaw flows," Physics of Fluids, vol. 20, no. 11, pp. 1-11, 2008.

[15] P. Daripa, "Hydrodynamic stability of multi-layer Hele-Shaw flows," Journal of Statistical Mechanics, vol. 12, pp. 1-32, 2008.

[16] D. R. Brouwer and J. D. Jansen, "Dynamic optimization of water flooding with smart wells using optimal control theory," in Proceedings of the SPE European Petroleum Conference, pp. 391-402, 2002.

[17] P. Sarma, K. Aziz, and L. J. Durlofsky, "Implementation of adjoint solution for optimal control of smart wells," in Proceedings of the SPE Reservoir Simulation Symposium, pp. 1-17, 2005. 
[18] L. L. Guo, S. R. Li, Y. B. Zhang, and Y. Lei, "Solution of optimal control of polymer flooding based on parallelization of iterative dynamic programming," Journal of China University of Petroleum, vol. 33, no. 3, pp. 167-174, 2009, (Edition of Natural Science).

[19] S. R. Li, Y. Lei, X. D. Zhang, and Q. Zhang, "Optimal control solving of polymer flooding based on a hybrid genetic algorithm," in Proceedings of the 29th Chinese Control Conference, pp. 5194-5198, 2010.

[20] Y. Lei, S. R. Li, and X. D. Zhang, "Optimal control solving of polymer flooding based on real-coded genetic algorithm," in Proceedings of the 8th World Congress on Intelligent Control and Automation, pp. 5111-5114, 2010.

[21] K. Aziz and A. Settari, Fundamentals of Reservoir Simulation, Elsevier Applied Science, New York, NY, USA, 1986.

[22] P. Sarma, W. H. Chen, L. J. Durlofsky, and K. Aziz, "Production optimization with adjoint models under nonlinear control-state path inequality constraints," in Proceedings of the SPE Intelligent Energy Conference and Exhibition, pp. 1-19, 2006.

[23] J. Nocedal and S. J. Wright, Numerical Optimization, Springer, New York, NY, USA, 2000. 


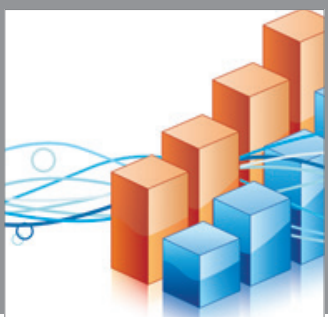

Advances in

Operations Research

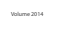

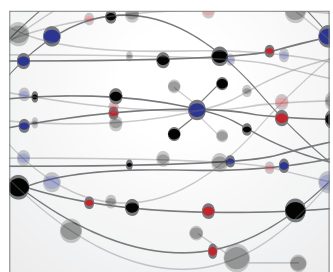

\section{The Scientific} World Journal
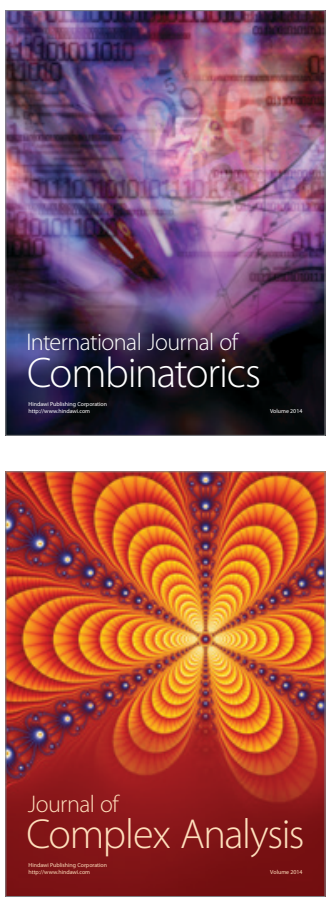

International Journal of

Mathematics and

Mathematical

Sciences
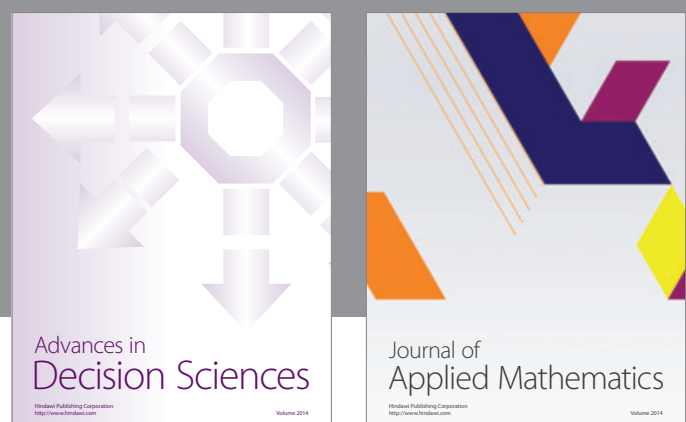

Journal of

Applied Mathematics
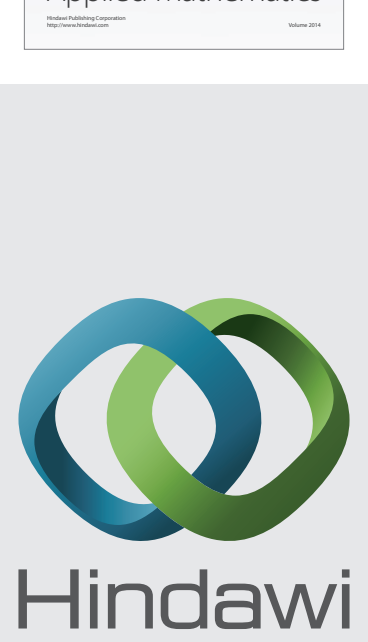

Submit your manuscripts at http://www.hindawi.com
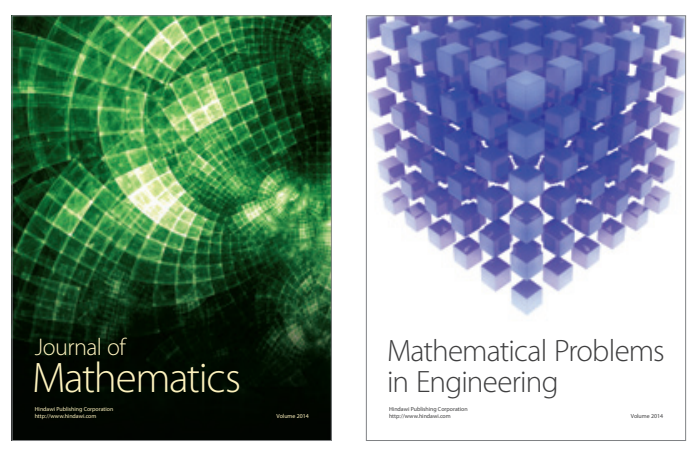

Mathematical Problems in Engineering
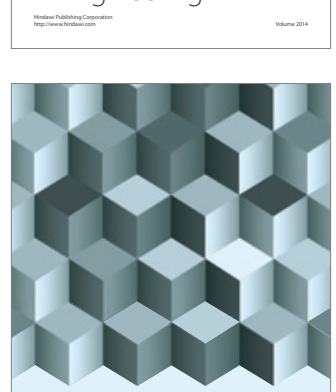

Journal of

Function Spaces
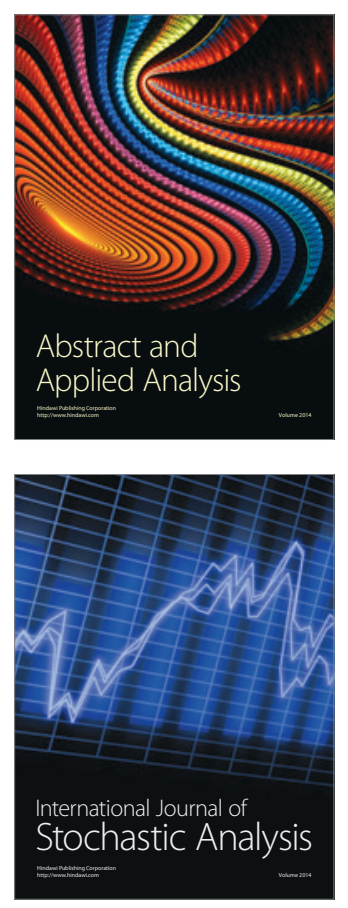

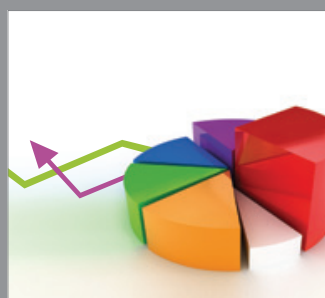

ournal of

Probability and Statistics

Promensencen
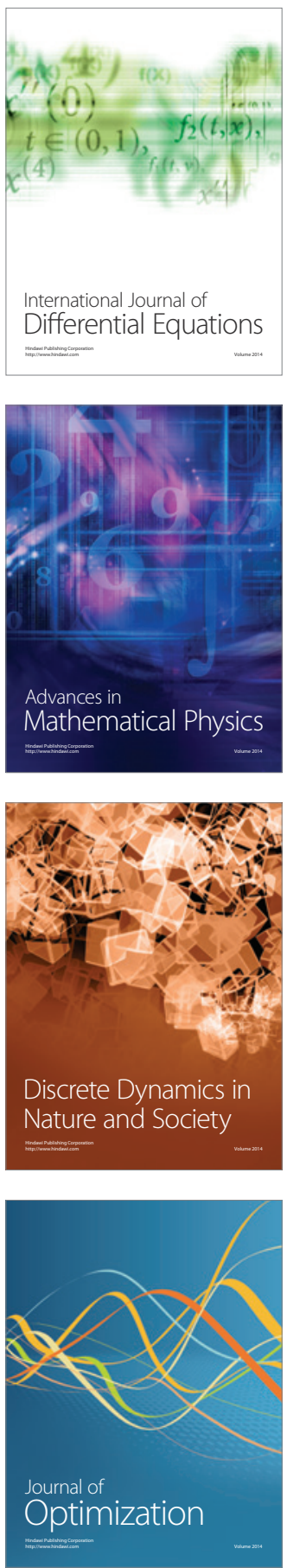